\section{Molecular classification of cutaneous malignant melanoma by gene expression profiling}

M. Bittner ${ }^{\star} \dagger$, P. Meltzer ${ }^{\star} \dagger$, Y. Chen ${ }^{\star}$, Y. Jiang ${ }^{\star}$, E. Seftor $\neq$, M. Hendrix $\neq$, M. Radmacher $\$$, R. Simon $\$$, Z. Yakhinill, A. Ben-Dor $\|$ g, N. Sampas $\|$, E. Dougherty\#, E. Wang ${ }^{2 / 2}$, F. Marincola ${ }^{*}$, C. Gooden ${ }^{\star}$, J. Lueders ${ }^{\star}$, A. Glatfelter ${ }^{\star}$, P. Pollock ${ }^{\star *}$, J. Carpten ${ }^{\star}$, E. Gillanders ${ }^{\star}$, D. Leja ${ }^{\star}$, K. Dietrich ${ }^{\star}$, C. Beaudry $\dagger \dagger$, M. Berens $\dagger \dagger$, D. Alberts $\ddagger \ddagger$, V. Sondak $\$ \S$, N. Hayward ${ }^{\star \star} \&$ J. Trent ${ }^{\star}$

* Cancer Genetics Branch, National Human Genome Research Institute, NIH, Bethesda, Maryland 20892, USA

$\ddagger$ Department of Anatomy and Cell Biology, University of Iowa Cancer Center, Iowa City, Iowa 52242-1109, USA

$\$$ National Cancer Institute, DCTDC, NIH, Bethesda, Maryland 20852, USA

$\|$ Chemical and Biological Systems Department, Agilent Laboratories, 3500 Deer Creek Road, Palo Alto, California 94304, USA

g Computer Science and Engineering Department, University of Washington, Seattle, Washington 98105, USA

\# Department of Electrical Engineering, Texas A \& M University, College Station, Texas 77843, USA

National Cancer Institute, Surgery Branch, NIH, Bethesda,

Maryland 20850, USA

${ }^{*}$ Queensland Institute of Medical Research, Herston, Queensland 4029, Australia

$\dagger \dagger$ Neuro-Oncology Laboratory, Barrow Neurological Institute, Phoenix, Arizona 85013-4496, USA

玤 Arizona Cancer Center, University of Arizona, Tucson, Arizona 85724, USA $\$ \$$ Department of Surgery, University of Michigan, Ann Arbor, Michigan 48109, USA

$\dagger$ These authors contributed equally to this work.

The most common human cancers are malignant neoplasms of the skin $^{1,2}$. Incidence of cutaneous melanoma is rising especially steeply, with minimal progress in non-surgical treatment of advanced disease ${ }^{3,4}$. Despite significant effort to identify independent predictors of melanoma outcome, no accepted histopathological, molecular or immunohistochemical marker defines subsets of this neoplasm ${ }^{2,3}$. Accordingly, though melanoma is thought to present with different 'taxonomic' forms, these are considered part of a continuous spectrum rather than discrete entities ${ }^{2}$. Here we report the discovery of a subset of melanomas identified by mathematical analysis of gene expression in a series of samples. Remarkably, many genes underlying the classification of this subset are differentially regulated in invasive melanomas that form primitive tubular networks in vitro, a feature of some highly aggressive metastatic melanomas ${ }^{5}$. Global transcript analysis can identify unrecognized subtypes of cutaneous melanoma and predict experimentally verifiable phenotypic characteristics that may be of importance to disease progression.

We, and others ${ }^{6-10}$, have proposed that a discrete and previously unrecognizable cancer taxonomy can be identified by viewing the systematized data from gene expression experiments. However, for melanoma, inherent or technically induced variation could obscure such a classification as its appearance is very similar between patient samples and, in contrast to haematologic cancers ${ }^{9,10}$, it has few known recurring genetic changes. To explore this question, we gathered expression profiles for 38 samples, including 31 melanomas and 7 controls (Table 1). We isolated total messenger RNA directly from melanoma biopsies or tumour cell cultures, prepared fluorescent complementary DNA from the message and hybridized them to a microarray containing probes for 8,150 cDNAs (representing 6,971 unique genes), obtaining quantitative and compara- tive measurements for each gene.

We compared the tumour cell mRNA with a single reference probe, providing normalized measures of the expression of each gene in each sample relative to the standard. Analysis of the normalized expression across all genes between samples provided a measure of the overall difference in expression pattern between samples. Similarly, the orthogonal analysis of linear covariance between pairs of genes across all samples provided a measure of the similarity of behaviour of the genes studied.

Figure 1 shows the integration of several analytical methods to visualize the overall expression pattern relationships between cutaneous melanoma tumour samples. Using a matrix of Pearson correlation coefficients from the complete pair-wise comparison of all experiments ${ }^{11}$, the 31 melanoma experiments are displayed as a hierarchical clustering dendrogram ${ }^{7,12}$ and as a three-dimensional multidimensional scaling (MDS) plot $^{7,13}$. The MDS plot displays the position of each tumour sample in three-dimensional Euclidean space, with the distance between experimental samples reflecting their approximate degree of correlation ${ }^{7,13}$. The analysis included all genes meeting a minimum level of expression in each hybridization. We also employed a non-hierarchical clustering algorithm (termed cluster affinity search technique; CAST $)^{14}$ to define experimental clusters. The resulting hierarchical dendrogram of the 31 melanoma samples (Fig. 1a) demonstrates that 19 samples are tightly clustered at the bottom of the dendrogram in the area of highest similarity. Likewise, the non-hierarchical CAST algorithm identified the identical major cluster 19 melanomas. This cluster is also a compact, readily separable grouping based on its overall similarity of expression pattern viewed by MDS (Fig. 1b). A three-dimensional animation of the MDS plot is presented in the Supplementary Information.

There is no single established method to estimate the significance of an observed degree of relationship obtained by cluster prediction techniques ${ }^{9,11}$. Accordingly, we used two independent approaches to test the validity of our cluster prediction of the 19-element cluster. The first approach (Fig. 1c) examines the power of individual genes to discriminate the major cluster of 19 from the remaining samples by examining the frequency of strong classifier genes compared to the expected frequency of such genes if expression is randomly variable, and to the frequency of strong classifiers in random partitions of the same samples into new groupings of 19 and 12. The non-randomness of the cluster results is evident. Specifically, many genes have expression patterns that differ strongly between the initial sample clusters and thus serve as good classifiers (Fig. 1c, red triangles). However, expression patterns are not readily found which classify the samples when they are grouped into random partitions of the same size (Fig. 1c, blue lines). Accordingly, in randomly formed clusters, expression behaviour is essentially indistinguishable from truly random behaviour of genes relative to these clusters (Fig. 1c, compare blue lines with open circles).

The second approach we used to test the validity of the cluster predictions is based on evaluating cluster membership after introducing random perturbations to the data set. For each sample, the log-ratio of each gene was perturbed by the introduction of random gaussian noise with the mean equal to 0 and the standard deviation equal to 0.15 (an estimate of variation derived by computing the median standard deviation of the log-ratios for single genes across all 31 samples). Hierarchical clustering was then performed on the perturbed data set and a comparison made between the original tree (Fig. 1a) and the perturbed tree. Comparisons involved cutting the original and perturbed trees into $k$ clusters followed by computing the proportion of paired samples clustering together in the original tree that did not cluster together in the perturbed tree (we refer to this measure as a weighted proportion of discrepant pairs because it gives more weight to larger clusters). The comparison was repeated over multiple perturbed data sets for each possible cut in the 
original tree $(k=2,3, \ldots, 30)$. For a given $k$, the weighted proportion of discrepant pairs was then averaged over the perturbed data sets resulting in the identification of weighted average discrepant pairs ( $\mathrm{WADP}_{k}$; see Supplementary Information).

Clusters that result from cutting the original tree into 9 or fewer groups are very reproducible (Fig. 1d). It is noteworthy that the rise in $\mathrm{WADP}_{k}$ almost exactly coincides with the division of the major 19-element cluster into smaller sub-clusters. These results strongly support the view that the major cluster of melanoma samples identified in this study represents a bona fide and highly reproducible grouping.
We then performed statistical tests to determine whether any clinical or tumour cell characteristics were specifically associated with the clustered group. Tests for associations between the major cluster of 19 samples and the remaining 12 melanoma samples were performed for several in vivo variables, including sex, age, biopsy site, Breslow thickness, Clark's level and survival. There was no statistically significant association between the cluster group and any clinical variable. There were also no significant associations with the in vitro variables, including $\mathrm{p} 16$ or $\beta$-catenin mutation status, in vitro pigmentation and cell passage number (see Supplementary Information).

a

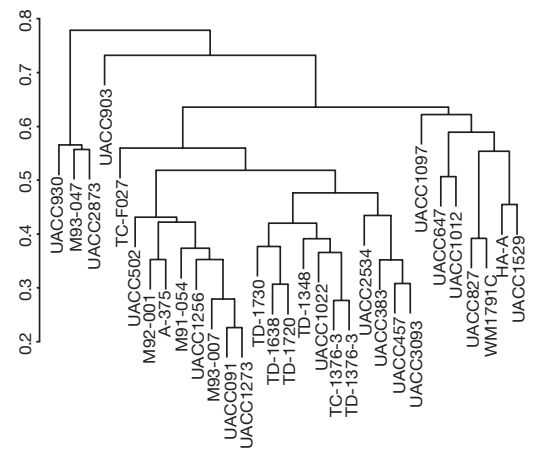

Figure 1 Clustering of gene expression data. a, Hierarchical clustering dendrogram with the cluster of 19 melanomas at the centre. $\mathbf{b}$, MDS three-dimensional plot of all 31 cutaneous melanoma samples showing major cluster of 19 samples (blue, within cylinder), and remaining 12 samples (gold). See Supplementary Information to view a three-dimensional animation. c, A plot of the observed and expected number of genes producing a given number of classification errors for a partition of the 31 melanomas into c

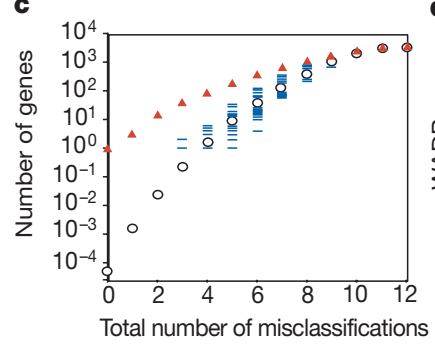

d

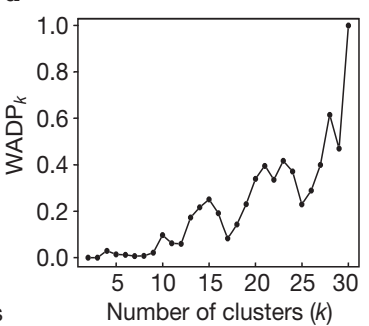

a

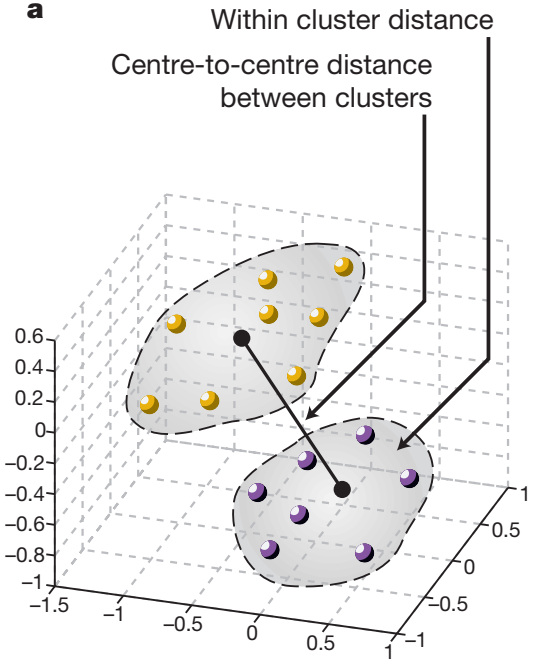

b $\varangle$
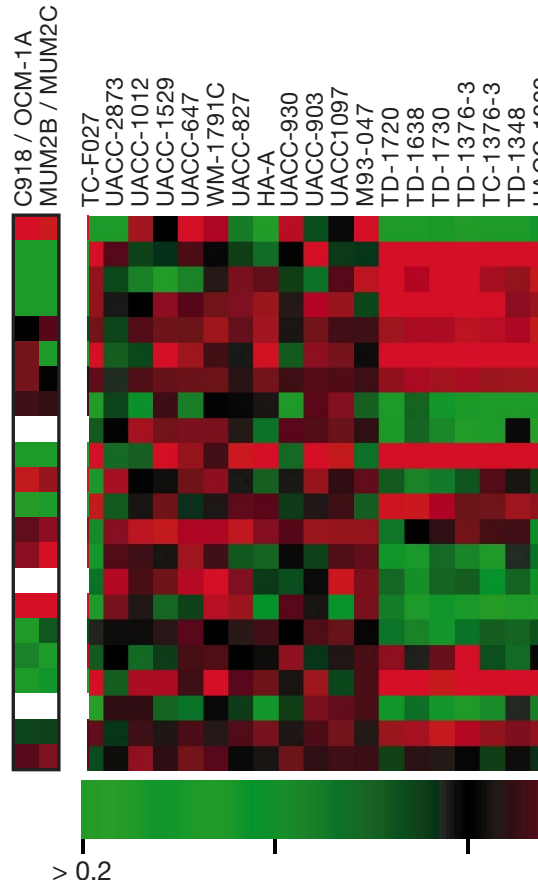

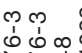

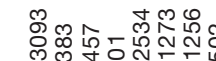

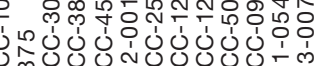
clusters; open circles, predicted results for randomly variable gene expression. d, Introduction of random gaussian noise followed by cuts from the top of the original tree (resulting in $k$ clusters), to determine discrepant pairs after perturbation (see Supplementary Information).
Figure 2 Identifying genes which discriminate melanoma clusters. a, MDS analysis ranking genes according to their impact on minimizing cluster volume and maximizing centre-to-centre inter-cluster distance. $\mathbf{b}$, Top 22 genes obtained by these criteria listed in order of decreasing weight (for a full list see Supplementary Information). Right, data from cutaneous melanomas identified on the horizontal axis and sorted by cluster

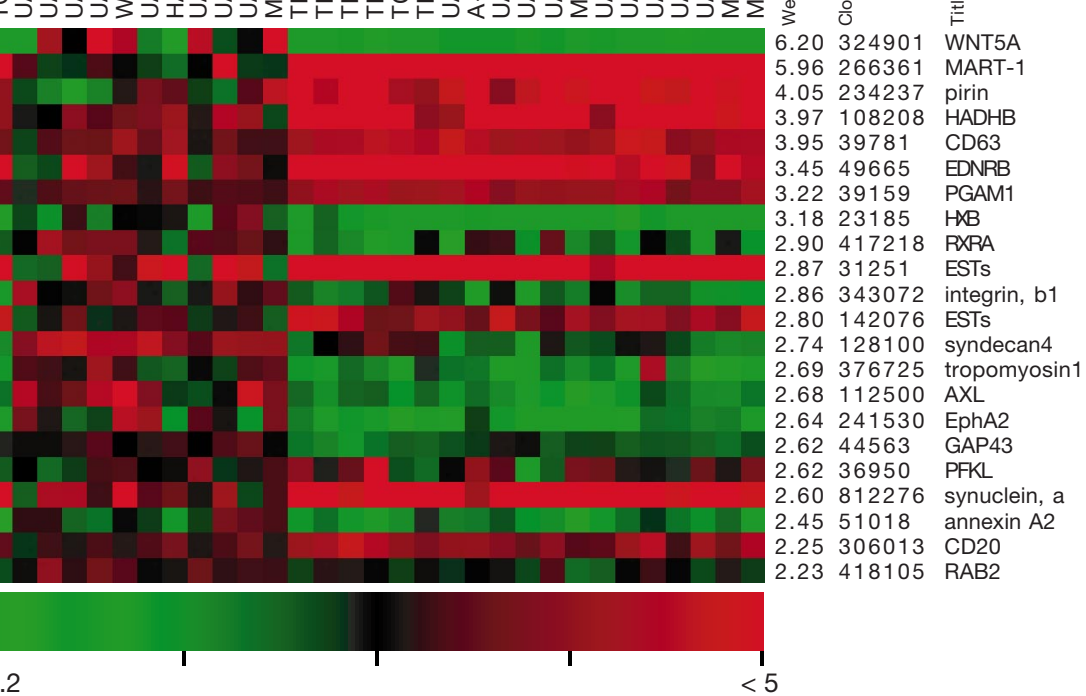

(described in ref. 5). Left, data from uveal melanomas expressed as the ratio of highly invasive to less invasive. Red, high ratios; green, low ratios (intensity of saturation scaled according to the ratio). The three genes not scored in the uveal samples were not included in the print design of the cutaneous samples. 
We included two pairs of specimens derived from the same patient in this sample set. These are M92-001 and M93-007 (two different samples from the same individual, surgically removed one year apart), and TD-1376-3 and TC-1376-3 (the biopsy sample and a cell culture of the same tumour carried three passages in vitro). Although there was no significant association between cell passage number and cluster group $(P=0.857$, see Supplementary Information), the TD-1376-3/TC-1376-3 pair were included to serve as another control for the effects of cell culture. Remarkably, of the 465 pairwise comparisons among the melanoma samples, the pairs TD1376-3/TC-1376-3 and M92-001/M93-007 are the second and third most highly correlated pairs of samples, with nearly identical correlation coefficients (Fig. 1b).

On the basis of the linear correlation of global gene expression in Fig. 1, Figs 2 and 3 illustrate the approach we have used to guide 'gene cluster' interpretation empirically. Fig. 2a depicts our statistical method for extracting a 'weighted list' of individual genes whose variance of change across all experiments correctly defines the boundary of a given sample cluster (for details see Supplemen- tary Information). Fig. 2b displays the list of genes with the most power to define the major melanoma cluster of 19 samples (Fig. 1a and $b$ ) in rank order along the vertical axis. The samples are ordered along the horizontal axis by cluster inclusion, and data are presented graphically as coloured images with the colour saturation directly proportional to the magnitude of the measured gene expression ratio (brightest red, highest $R / G$ ratio; black squares, $R / G$ ratio $=1$; brightest greens, lowest $R / G$ ratio). The complete list of genes discriminating the major cluster is in the Supplementary Information.

The weighted gene list can also be used to guide analysis of the larger gene expression data set. Figure 3a displays all data from the cutaneous melanoma samples in this study as a coloured image with genes ordered along the vertical axis by similarity of expression pattern (after ref. 12). However, rather than basing analysis of this large ( $>300,000$ elements) data set entirely on visual selection, we used genes from the weighted list to index gene cluster selection. Figure $3 \mathrm{~b}-\mathrm{e}$ illustrates this approach using four genes from the 'weighted list' in Fig. 2b (MART-1, CD63, tropomyosin and

\begin{tabular}{|c|c|c|c|c|c|c|c|c|c|}
\hline Case no. & Sex/Age & Biopsy site & $\begin{array}{c}\text { Passage no. } \\
\text { (Biopsy) }\end{array}$ & $\begin{array}{l}\text { p16 mutation } \\
\text { status }^{*}\end{array}$ & $\begin{array}{l}\text { Invasive } \\
\text { ability† }\end{array}$ & $\begin{array}{l}\text { Vasulogenic } \\
\text { mimicry } \ddagger\end{array}$ & $\begin{array}{c}\text { Gel } \\
\text { contraction§ }\end{array}$ & $\begin{array}{c}\text { Cell } \\
\text { motilityll }\end{array}$ & $\begin{array}{c}\text { Scratch } \\
\text { wound } \\
(\%) \emptyset\end{array}$ \\
\hline \multicolumn{10}{|c|}{ Melanoma primary cluster } \\
\hline UACC-502 & $\mathrm{M} / 69$ & Cervical node & 3 & Deleted & $2.8 \pm 0.1 \%$ & - & ND & ND & 37 \\
\hline M92-001 & $\mathrm{F} / 43$ & Ankle & 2 & Deleted & $3.0 \pm 0.5 \%$ & - & ND & $76.80 \pm 2.96$ & 22 \\
\hline A-375 & $\mathrm{F} / 54$ & Skin & ND & Mutation & $2.8 \pm 0.2 \%$ & - & ND & $67.80 \pm 4.40$ & 26 \\
\hline M91-054\# & $\mathrm{M} / 45$ & Axill, lymph node & 3 & WT & \# & $\#$ & $\#$ & ND & 30 \\
\hline UACC-1256 & $\mathrm{F} / 67$ & Thigh femoral node & 9 & Deleted & ND & ND & ND & ND & ND \\
\hline M93-007 & $\mathrm{F} / 43$ & Ankle & 3 & Deleted & $2.6 \pm 0.1 \%$ & - & - & ND & 12 \\
\hline UACC-091 & $\mathrm{M} / 52$ & Unk & 7 & Deleted & $2.1 \pm 0.2 \%$ & - & - & ND & 11 \\
\hline UACC-1273 & $\mathrm{M} / 50$ & Axill. lymph node & 16 & Mutation & $2.5 \pm 0.3 \%$ & - & - & ND & 13 \\
\hline TD-1730 & $\mathrm{M} / 55$ & Thyroid lobe & Biopsy & ND & ND & ND & ND & ND & ND \\
\hline TD-1638 & $\mathrm{M} / 49$ & Paraspinous & Biopsy & ND & ND & ND & ND & ND & ND \\
\hline TD-1720 & $\mathrm{M} / 29$ & Shoulder & Biopsy & ND & ND & ND & ND & ND & ND \\
\hline TD-1348 & $\mathrm{M} / 44$ & Axill. lymph node & Biopsy & ND & ND & ND & ND & ND & ND \\
\hline UACC-1022 & $\mathrm{F} / 53$ & Chest wall & 13 & WT & $2.9 \pm 0.1 \%$ & - & - & ND & 63 \\
\hline TC- $1376^{\text {th }}$ & $\mathrm{M} / 30$ & Distal ileum & 3 & ND & ND & ND & ND & ND & 21 \\
\hline TD-1376 & $\mathrm{M} / 30$ & Distal ileum & Biopsy & ND & ND & ND & ND & ND & ND \\
\hline UACC-2534 & $\mathrm{M} / 68$ & Abdomen & 7 & Deleted & $3.2 \pm 0.02 \%$ & - & ND & ND & 7 \\
\hline UACC-383 & $\mathrm{M} / 69$ & Thigh femoral node & 29 & Deleted & $2.3 \pm 0.2 \%$ & - & ND & $70.40 \pm 5.27$ & 35 \\
\hline UACC-457 & FUkn & Unk & 19 & WT & $3.1 \pm 0.2 \%$ & - & ND & $12.80 \pm 0.05$ & ND \\
\hline UACC-3093 & $\mathrm{M} / 75$ & Axill. lymph node & 4 & WT & ND & ND & ND & $40.30 \pm 2.00$ & 24 \\
\hline \multicolumn{10}{|c|}{ Melonoma non-clustered } \\
\hline UACC-930 & $\mathrm{F} / 35$ & Sm. bowel & 4 & WT & $4.8 \pm 0.3 \%$ & \pm & - & ND & 50 \\
\hline M93-047 & $\mathrm{F} / 75$ & Axill. Iymph node & 3 & Mutation & $10.7 \pm 0.03 \%$ & + & + & ND & 75 \\
\hline UACC-2973 & $\mathrm{M} / 37$ & Axill. lymph node & 5 & ND & ND & ND & ND & ND & 48 \\
\hline UACC-903 & $\mathrm{M} / 25$ & Back & 14 & Deleted & $3.8 \pm 0.3 \%$ & + & - & ND & 91 \\
\hline TC-FO27 & $\mathrm{M} / 30$ & Rt. chest wall & 6 & ND & ND & ND & ND & ND & 91 \\
\hline UACC-1097 & $\mathrm{M} / 56$ & Rectus muscle & 6 & Mutation & ND & ND & ND & ND & 34 \\
\hline UACC- $647^{\star *}$ & $\mathrm{M} / 32$ & Axill. node & 14 & WT & $3.8 \pm 0.1 \%$ & + & \pm & ND & 55 \\
\hline UACC-1012 & $\mathrm{M} / 54$ & Neck & 3 & ND & $4.9 \pm 0.1 \%$ & ND & $\overline{N D}$ & $122.00 \pm 11.30$ & 54 \\
\hline UACC-827 & $\mathrm{F} / 32$ & Rt. breast & 16 & Mutation & ND & ND & ND & ND & 32 \\
\hline WM1791C & Unk & Ukn & 52 & ND & $4.6 \pm 0.3 \%$ & + & ND & $141.00 \pm 11.40$ & 71 \\
\hline $\mathrm{HA}-\mathrm{A}$ & F/Ukn & Ukn & 19 & ND & $3.9 \pm 0.5 \%$ & \pm & ND & $211.00 \pm 12.40$ & 62 \\
\hline UACC-1529 & $\mathrm{M} / 48$ & Axill. lymph node & 13 & Mutation & $4.2 \pm 0.5 \%$ & + & - & ND & ND \\
\hline \multicolumn{10}{|c|}{ Uveal melanoma samples } \\
\hline OCM-1A & Unk & Primary & 25 & ND & $2.2 \pm 0.1 \%$ & - & _ & ND & ND \\
\hline C918 & $\mathrm{F} / 60$ & Primary & 15 & ND & $12.9 \pm 0.3 \%$ & + & + & ND & ND \\
\hline MUM-2C & $\mathrm{M}$ & Liver metastasis & 8 & ND & $2.0 \pm 0.1 \%$ & - & - & ND & ND \\
\hline MUM-2B & M & Liver metastasis & 8 & ND & $13.3 \pm 0.6 \%$ & + & + & ND & ND \\
\hline
\end{tabular}

Control samples

Nil. C (fibroblast); UACC-3149 (ovarian adenocarcinoma); MCF-10A (breast epithelium); CRL-1634 (fibroblast); SRS-3 (cell culture variant); SRS-5 (cell culture variant); RMS-13 (rhabdomyosarcoma)

* Mutation status of indicated samples for 16 obtained by sequencing. Deleted, homozygous. Supplementary Information includes the specific mutations in 16 for each sample tested. Samples were also sequenced for $\beta$-catenin. No example of $\beta$-catenin mutation was observed.

† Ability to invade a defined basement matrix. $P=0.0055$; $t$-test for two populations.

¥ Tube forming ability at 5 days in a three-dimensional matrigel matrix.

$\S$ Ability to contract floating collagen I gels at 5 days as compared to HT-1080 fibrosarcoma cells

$\|$ Migration rates expressed in $\mu \mathrm{m}$ per day. Mean from eight experiments \pm s.d. ( $P=0.0063 ; t$-test for two populations). Rates below $100 \mu \mathrm{m}$ per day completely segregates in the melanoma primary cluster. I Ability to close in vitro scratch wound at $24 \mathrm{~h}$. Photographs of the wound were measured and percentage wound closure determined ${ }^{23}(P<0.00002, t$-test for two populations).

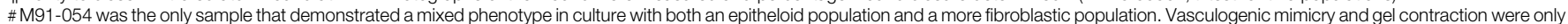
observed in the epitheloid population. Scratch assay resulted in $30 \%$ closure after $24 \mathrm{~h}$ for both populations.

t TC-1376 mRNA was isolated after short term ( 3 passage) culture of the biopsy sample from the patient TD-1376 allowing the effects of short term culture on the expression profile to be observed. ** UACC-647 cells form extensive cord-like networks by 5 days. 


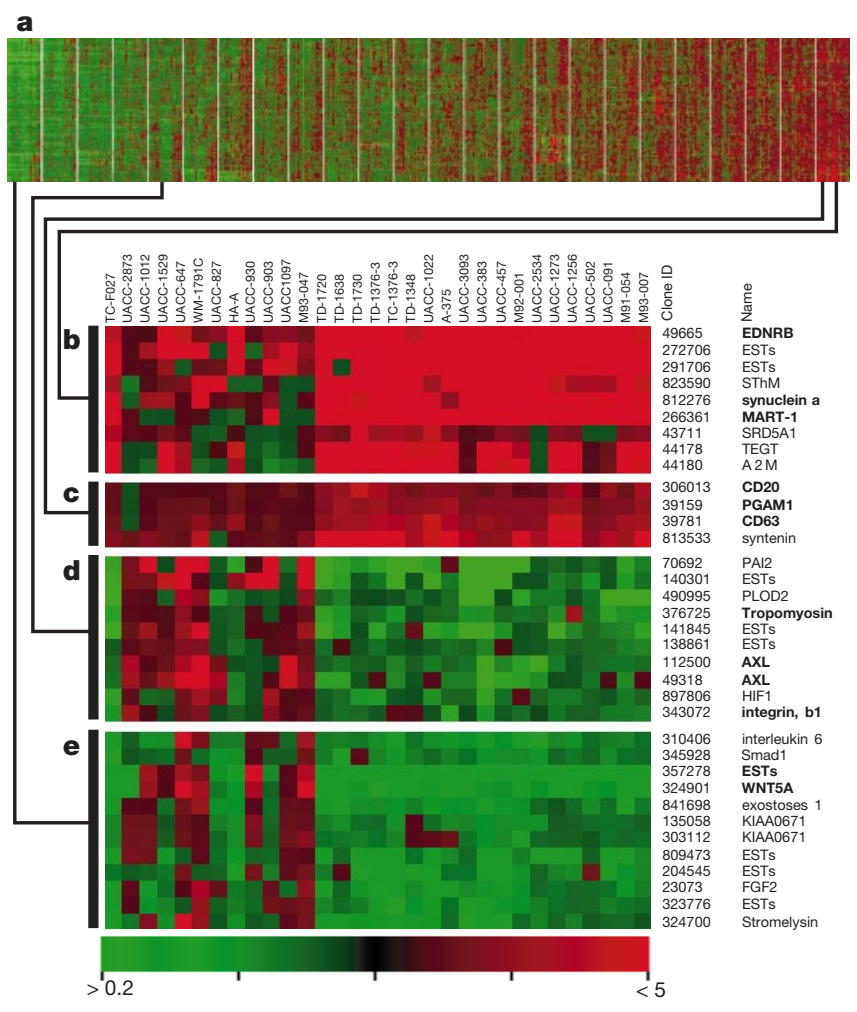

Figure 3 Guiding gene cluster selection. a, Two-dimensional cluster analysis of cutaneous melanoma samples (horizontal axis) and genes (vertical axis, presented in segments). b-e, Data from a queried at regions corresponding to four top discriminators of the major cluster: MART-1 (b), CD63 (c), tropomyosin (d) and WNT5A (e). Note that these clusters include other genes from the discriminator list (bold). The major cluster of 19 samples is visually apparent on the left of this display. The full list of gene names and corresponding calculated ratio information is provided in the Supplementary Information.

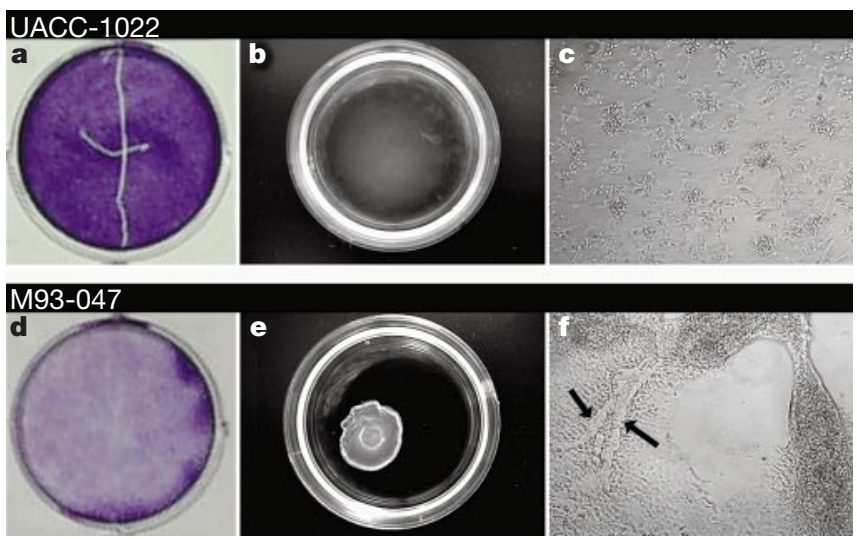

Figure 4 Variation in biological properties of melanoma clusters. a-c, A representative member of the major melanoma cluster (UACC-1022). $\mathbf{d}-\mathbf{f}$, A sample falling outside of the major cluster (M93-047). The two groups differ in the ability to migrate into a scratch wound $(\mathbf{a}, \mathbf{d})$, contract collagen gels $(\mathbf{b}, \mathbf{e})$ and form tubular networks $(\mathbf{c}, \mathbf{f})$. Results of these and additional cell mobility/invasion assays are included in Table 1. Tubular network formation (vasculogenic mimicry ${ }^{5}, \mathbf{f}$ ) and collagen gel contraction (related to the patterning of vascular channels, e) were observed only outside the major cluster (Table 1).
WNT5A), to interrogate the entire gene expression data set represented in Fig. 3a.

Finally, in parallel to our microarray analysis of cutaneous melanoma, we studied a series of uveal melanoma specimens characterized for properties related to metastasis, including invasive ability and vasculogenic mimicry in vitro ${ }^{5}$. These samples were hybridized pairwise, directly comparing highly invasive cells to their less invasive counterparts. We examined the pattern of gene expression in these phenotypically characterized cells with respect to the weighted discriminator list (Fig. 2b) that defines the major cluster of 19 cutaneous melanomas. Strikingly, genes expressed in common in the highly invasive uveal melanoma cells (Fig. 2b, inset) were strongly anti-correlated with the same gene from the major cluster of cutaneous melanoma samples (Fig. 2b). This observation, coupled with the known biological function of genes within the weighted list, indicated that specimens assigned within the major cutaneous melanoma cluster (Fig. 1a, b) would have reduced motility and reduced invasive ability as they have downregulation of genes related to cell spreading or migration, including formation of focal adhesions ${ }^{15,16}$. Specific genes with reduced expression in the major cluster included integrin $\beta 1$ (refs 17,18 ), integrin $\beta 3$ (ref. 19), integrin $\alpha 1$ (ref. 18), syndecan 4 (ref. 20) and vinculin ${ }^{21}$ (Figs 2 and 3; see Supplementary Information). In samples outside the major cluster increased expression of fibronectin is particularly interesting. With other reports $^{22,23}$, this observation indicates that these cells are induced to secrete this pro-migratory molecule, consistent with an important role for focal contacts in modulating melanoma cell motility.

We then directly tested the prediction from the array results that cell spreading and migration could be discordant between melanoma cluster groups. Cutaneous melanomas (assigned either in or out of the major cluster) were characterized using a series of cellular assays applied to test cell motility and invasiveness (Table 1, Fig. 4). Figure 4 illustrates the discordance of cutaneous melanoma samples within the major cluster and those outside this group. As predicted from the analysis of their gene expression patterns, melanomas within the major cluster had reduced motility $(P=0.0063)$, invasive ability $(P=0.0055)$ and vasculogenic mimicry in comparison with melanomas outside the major cluster (Table 1).

The patient population in this study had a uniformly poor prognosis, and neither typical clinical factors (for example, age, sex, biopsy site) nor in vitro characteristics (for example, passage number) provide strong correlation with clinical outcome, or expression information (see Supplementary Information). In contrast, molecular classification of these tumours on the basis of gene expression (Fig. 1, Table 1) could identify a previously undetected subtype of this cancer. The analyses described here were not designed to address the relationship of gene expression profile and clinical outcome in melanoma patients, and thus the clinical relevance of our observed subgrouping awaits further analysis. However, survival information was available on 15 patients, and the results, though not statistically significant, are of interest. Three deaths occurred out of 10 patients in the tight cluster of 19 while 4 deaths occurred out of 5 patients in the remaining group $(\log$-rank $P$-value $=0.135)$. Our results indicate melanoma will provide a unique opportunity to study a homogeneous group of patients to determine if gene expression patterns predict prognosis or therapeutic response in settings where we cannot currently determine who is most at risk for rapid disease progression and death.

Finally, classification of melanoma on the basis of gene expression patterns is possible, despite the prevailing view that the 'taxonomy' of this disease falls in a continuous spectrum lacking discernable entities ${ }^{2}$. Our data show that melanoma is a useful model to identify genes critical for aspects of the metastatic process, including tumour 
cell motility and the ability to form primitive tubular networks that may contribute to tumour perfusion. The extent to which melanoma samples can be clinically subdivided by expression patterns remains to be elucidated. However, our identification of genes 'weighted' for their ability to discriminate a subset of melanomas should provide a sound molecular basis for the dissection of other clinically relevant subsets of this tumour.

\section{Methods}

\section{Samples}

Cultured cells were collected and mRNA isolated as described (ref. 25; www.nhgri.nih.gov/DIR/microarray). Samples underwent a series of controls for quality of mRNA, labelling and hybridization, as well as sample integrity (including genotyping DNA from all samples with five dinucleotide markers from four different chromosomes to insure individuality). The entire coding sequence of the p16 gene and exon 3 of the $\beta$-catenin genes was sequenced to assess the mutation status of all available samples (see Supplementary Information). The biopsy tumour specimens used in this study were obtained with Institutional Review Board approval and clinical information is provided in the Supplementary Information. Biopsies were debrided, dissected into small pieces and frozen in liquid nitrogen. Frozen specimens were immediately placed into TRIzol Reagent (Gibco BRL), homogenized and mRNA isolated as described (ref. 25; www.nhgri.nih.gov/DIR/microarray).

\section{Microarrays}

The 8,150 human cDNAs used in this study were obtained under a Cooperative Research and Development Agreement with Research Genetics and 6,912 were verified by sequence. This set of cDNAs is part of a larger collection (refs 7, 24; www.nhgri.nih.gov/ DIR/microarray). On the basis of the Unigene build of 9 March 2000 (http:/I www.ncbi.nlm.nih.gov/UniGene/build.html), the 8,150 cDNAs represent 6,971 unique genes in this melanoma array. All clones were confirmed by resequencing if necessary. Microarrays were hybridized, scanned and image analysis performed as described (refs 7 , 25; www.nhgri.nih.gov/DIR/microarray).

\section{Statistical methods}

Detailed information on all statistical methods is in the Supplementary Information. Agglomerative hierarchical clustering of the 31 melanomas on the basis of their gene expression profiles was performed as described ${ }^{7,11}$, to investigate relationships between tumour samples. Average linkage was used, as well as a dissimilarity measure of one minus the Pearson correlation coefficient of log ratios. The cutoff employed to obtain the observed partitioning was 0.54 . The MDS was performed using an implementation of MDS in the MATLAB package. A non-hierarchical clustering algorithm ${ }^{14}$ was used to define experimental clusters. This approach takes a graph theoretic approach, and makes no assumptions on the similarity function or the number of clusters sought.

To generate the weighted gene list, cluster compaction and separation were evaluated. For a given clustering result, $n_{1}=19$ and $n_{2}=12$, the discriminative weight of each gene $w=d_{\mathrm{B}} /\left(k_{1} d_{w 1}+k_{2} d_{w 2}+\alpha\right)$; where $d_{\mathrm{B}}$ is the centre-to-centre distance (between cluster Euclidean distance), $d_{w i}$ is the average Euclidean distance among all sample pairs within cluster $i, k_{i}=t_{i} /\left(t_{1}+t_{2}\right)$ for a total of $t_{i}$ sample pairs in cluster $i$, and $\alpha$ is a small constant ( 0.1 in our study) to prevent the zero denominator case (Fig. 2a). Genes may then be ranked on the basis of $w$.

\section{In vitro biological assays}

Floating collagen lattices were prepared and used to test selected cell lines for their ability to deform the gels as described (ref. 5; Table 1 legend). Samples were also tested for their ability to migrate into an in vitro scratch wound as described ${ }^{26}$. Cells were stained with Giemsa, a digital micrograph of the region was prepared and the stained area as a percent of total area in the scraped and open sub-regions was estimated by a thresholding procedure using IPLabs Spectrum (Scanalytics, Vienna, Virginia) software. Results in Table 1 represent data from $24 \mathrm{~h}$ after plating on coverslips treated with fibronectin (FN; $10 \mu \mathrm{g} \mathrm{ml}^{-1}$; ref. 26).

Examples of tubular network formation (associated with vasculogenic mimicry) could be observed following seeding of cell lines onto three-dimensional gels of polymerized Matrigel or Type 1 collagen (Collaborative Biochemical) as described (ref. 5; Table 1).

Table 1 lists results from high throughput screening for cell migration as the radial dispersion of cells from an initial confluent monolayer of 2,000 melanoma cells deposited within a $1.0 \mathrm{~mm}$ circular area on glass surfaces precoated with FN $\left(100 \mu \mathrm{g} \mathrm{ml}^{-1}\right.$; refs 27 , 28).

Selected cell lines were tested for their ability to invade a defined basement membrane matrix. Tumour cells $\left(1 \times 10^{5}\right)$ were seeded into the upper wells of the membrane invasion culture system (MICS) chamber $^{29}$ onto collagen/laminin/gelatincoated (Sigma) polycarbonate membranes containing $10-\mu \mathrm{m}$ pores (Osmonics, Livermore, California) containing $1 \times$ Mito+ Serum Extender (Becton Dickinson). After $24 \mathrm{~h}$ of incubation at $37^{\circ} \mathrm{C}$, the cells that invaded each membrane were collected, stained and counted as described ${ }^{30}$. Percent invasion was corrected for proliferation and calculated as (total number of invading cells/ total number of cells seeded) $\times 100$.
Received 12 January; accepted 16 May 2000.

1. Hall, H. I., Miller, D. R., Rogers, J. D. \& Bewerse, B. Update on the incidence and mortality from melanoma in the United States. J. Am. Acad. Dermatol. 40, 35-42 (1999).

2. Weyers, W., Euler, M., Diaz-Cascajo, C., Schill, W. B. \& Bonczkowitz, M. Classification of cutaneous malignant melanoma: a reassessment of histopathologic criteria for the distinction of different types. Cancer 86, 288-299 (1999).

3. Byers, H. R. \& Bhawan, J. Pathologic parameters in the diagnosis and prognosis of primary cutaneous melanoma. Hematol. Oncol. Clin. North Am. 12, 717-735 (1998).

4. McMasters, K. M., Sondak, V. K., Lotze, M. T. \& Ross, M. I. Recent advances in melanoma staging and therapy. Ann. Surg. Oncol. 6, 467-475, (1999).

5. Maniotis, A. J. et al. Vascular channel formation by human melanoma cells in vivo and in vitro: vasculogenic mimicry. Am. J. Pathol. 155, 739-752 (1999).

6. DeRisi, J. et al. Use of a cDNA microarray to analyse gene expression patterns in human cancer. Nature Genet. 14, 457-460 (1996).

7. Khan, J. et al. Gene expression profiling of alveolar rhabdomyosarcoma with cDNA microarrays. Cancer Res. 58, 5009-5013 (1998)

8. Perou, C. M. et al. Distinctive gene expression patterns in human mammary epithelial cells and breast cancers. Proc. Natl Acad. Sci. USA 96, 9212-9217 (1999).

9. Golub, T. R. et al. Molecular classification of cancer: class discovery and class prediction by gene expression monitoring. Science 286, 531-537, (1999).

10. Alizadeh, A. et al. Distinct types of diffuse large B-cell lymphoma identified by gene expression profiling. Nature 403, 503-511 (2000).

11. Bittner, M., Meltzer P. \& Trent J. Data analysis and integration: of steps and arrows. Nature Genet. 22, 213-215 (1999).

12. Eisen, M. B., Spellman, P. T., Brown P. O. \& Botstein, D. Cluster analysis and display of genome-wide expression patterns. Proc. Natl Acad. Sci. USA 95, 14863-14868 (1998).

13. Everitt, B. Applied Multivariate Data Analysis. (Oxford Univ. Press, New York, 1992)

14. Ben-Dor, A., Shamir, R. \& Yakhini, Z. Clustering gene expression patterns. J. Comput. Biol. 6, 281-297 (1999).

15. Adams, J. C. Characterization of cell-matrix adhesion requirements for the formation of fascin microspikes. Mol. Biol. Cell 8, 2345-2363 (1997).

16. Scott, G. \& Liang, H. pp125FAK in human melanocytes and melanoma: expression and phosphorylation. Exp. Cell Res. 219, 197-203 (1995).

17. Jannji, B., Melchior, C., Guon, V., Vallar, L. \& Kieffer, N. Autocrine TGF-beta-regulated expression of adhesion receptors and integrin-llinked kinase in HT-144 melanoma cells correlates with their metastatic phenotype. Int. J. Cancer 83, 255-262 (1999).

18. Hieken, T. et al. Betal integrin expression in malignant melanoma predicts occult lymph note metastases. Surgery 118, 669-673 (1995).

19. Van Belle, P. et al. Progression-related expression of beta3 integrin in melanomas and nevi. Hum. Pathol. 30, 562-567 (1999)

20. Woods, A., Longley, R., Tumova, S. \& Couchman, J. Syndecan-4 binding to the high affinity heparinbinding domain of fibronectin drives focal adhesion formation in fibroblasts. Arch. Biochem. Biophys. 374, 66-72 (2000)

21. Helige, C. et al. Interrelation of motility, cytoskeletal organization and gap junctional communication with invasiveness of melanocytic cells in vitro. Invasion Metastasis 17, 26-41 (1997).

22. Maung, K., Easty, DJ., Hill, S. \& Bennett, D. Requirement for focal adhesion kinase in tumor cell adhesion. Oncogene 18, 6824-6828 (1999).

23. Silletti, S., Paku, S. \& Raz, A. Autocrine motility factor and the extracellular matrix. I. Coordinate regulation of melanoma cell adhesion, spreading and migration involves focal contact reorganization. Int. J. Cancer 76, 120-128 (1998)

24. Duggan, D. J., Bittner, M., Chen, Y., Meltzer, P. \& Trent, J. Expression profiling using cDNA microarrays. Nature Genet. 21, 10-14, (1999).

25. Khan, J., Bittner, M., Chen, Y., Meltzer, P. \& Trent, J. DNA Microarray technology: the anticipated impact on the study of human disease. Biochim. Biophys. Acta 1423, 17-28 (1999).

26. Tamura, M. et al. Inhibition of cell migration, spreading, and focal adhesions by tumor suppressor PTEN. Science 280, 1614-1617 (1998).

27. Berens, M., Rief, M., Loo, M. \& Giese, A. The role of extracellular matrix in human astrocytoma migration and proliferation studied in a microliter scale assay. Clin. Exp. Metastasis 12, 405-415 (1994).

28. Giese, A., Loo, M., Norman, S., Treasurywala, S. \& Berens, M. Contrasting migratory response of astrocytoma cells to tenascin mediated by different integrins. J. Cell Sci. 109, 2161-8 (1996).

29. Hendrix, M., Seftor, E., Seftor, R. \& Fidler, I. A simple quantitative assay for studying the invasive potential of high and low human metastatic variants. Cancer Lett. 38, 137-147 (1987).

30. Hendrix, M., Seftor, E., Chu, Y., Trevor, K. \& Seftor, R. Role of intermediate filaments in migration, invasion and metastasis. Cancer Metastasis Rev. 15, 507-525 (1996)

Supplementary information is available on Nature's World-Wide Web site (http:// nature.com) or as paper copy from the London editorial office of Nature.

\section{Acknowledgements}

Grant support from NIH (M.E.B, M.H.) and the Australian National Health and Medical Research Council (N.H.). We thank D. Edwards for access to instrumentation used in high throughput cell migration screening; A. Cress, K. Yamada and P. Schwartzberg for discussions on cell motility and assay design; and J. Pe'er, R. Folberg, K. Daniels and J. Kan-Mitchell for donation of uveal melanoma cell lines described in ref. 5 .

Correspondence and requests for materials should be addressed to J.T. (jtrent@nih.gov) or M.B. (mbittner@nhgri.nih.gov). 